European J. Combin. 70 (2018), 317-324. MR3779621.

doi: $10.1016 /$ j.ejc. 2018.01 .003

\title{
Overpartitions with bounded part differences
}

\author{
Shane Chern and Ae Ja Yee
}

\begin{abstract}
We generalize recent results of Breuer and Kronholm, and Chern on partitions and overpartitions with bounded differences between largest and smallest parts. We prove our generalization both analytically and combinatorially.

Keywords. Partitions, overpartitions, bounded difference between largest and smallest parts, combinatorial proof.

2010MSC. Primary 05A17; Secondary 05A19, 11P84.
\end{abstract}

\section{Introduction}

A partition of a positive integer $n$ is a non-increasing sequence of positive integers whose sum equals $n$. For example, there are three partitions of 3: $3,2+1$, and $1+1+1$.

In [2], Andrews, Beck and Robbins explored partitions with the difference between largest and smallest parts equal to $t$ for some positive integer $t$. Motivated by their work, Breuer and Kronholm [3] studied the number of partitions of $n$ with the difference between largest and smallest parts bounded by $t$, and they showed that the generating function is

$$
\sum_{n \geq 1} p_{t}(n) q^{n}=\frac{1}{1-q^{t}}\left(\frac{1}{(q)_{t}}-1\right)
$$

where $p_{t}(n)$ counts the number of partitions of $n$ with the difference between largest and smallest parts bounded by $t$, and

$$
(a)_{n}=(a ; q)_{n}:=\prod_{k=0}^{n-1}\left(1-a q^{k}\right) .
$$

The proof of Breuer and Kronholm has a geometric flavour, and their main tool used in the proof is polyhedral cones. Subsequently, Chapman [4] also provided a simpler proof, which involves $q$-series manipulations.

An overpartition of $n$ is a partition of $n$ in which the first occurrence of each distinct part may be overlined. For example, there are eight overpartitions of 3: 3 , $\overline{3}, 2+1, \overline{2}+1,2+\overline{1}, \overline{2}+\overline{1}, 1+1+1$, and $\overline{1}+1+1$.

Recently, motivated by the works of Andrews, Beck and Robbins, Breuer and Kronholm, and Chapman, the first author [5] considered an overpartition analogue with bounded difference between largest and smallest parts.

To obtain a generating function analogous to (1.1), apart from requiring the difference between largest and smallest parts less than or equal to $t$, the first author added the following restriction: if the difference between largest and smallest parts 
is exactly $t$, then the largest part cannot be overlined. Let $g_{t}(n)$ count the number of such overpartitions of $n$. Then it was shown that

$$
\sum_{n \geq 1} g_{t}(n) q^{n}=\frac{1}{1-q^{t}}\left(\frac{(-q)_{t}}{(q)_{t}}-1\right) .
$$

The first proof in his paper uses heavy $q$-series manipulation, which originates from [2]. His second proof, which consists of many combinatorial ingredients such as the overpartition analogue of $q$-binomial coefficients introduced by Dousse and Kim [6], however, still needs some nontrivial computation, and hence it is not completely combinatorial.

The main purpose of this paper is to provide a completely combinatorial and transparent proof of (1.2). More precisely, we prove the following refined result.

Theorem 1.1. For a positive integer $t$, let $g_{t}(m, n)$ count the number of overpartitions of $n$ in which there are exactly $m$ overlined parts, the difference between largest and smallest parts is at most $t$, and if the difference between largest and smallest parts is exactly $t$, then the largest part cannot be overlined. Then

$$
\sum_{n \geq 1} \sum_{m \geq 0} g_{t}(m, n) z^{m} q^{n}=\frac{1}{1-q^{t}}\left(\frac{(-z q)_{t}}{(q)_{t}}-1\right) .
$$

We remark that (1.1) and (1.2) follow immediately from (1.3) by taking $z \rightarrow 0$ and $z \rightarrow 1$, respectively.

1.1. Notation and terminology. Throughout this paper, $\mathbb{Z}_{\geq 0}$ and $\mathbb{Z}_{>0}$ denote the set of nonnegative integers and positive integers, respectively. Given a partition or an overpartition $\lambda$ of $n$, let $\ell(\lambda)$ be the number of parts of $\lambda$ and $|\lambda|=n$ be the sum of the parts of $\lambda$. When $\lambda$ is an overpartition, we use $o(\lambda)$ to count the number of overlined parts in $\lambda$. We write parts in weakly decreasing order.

For a positive integer $t$, we denote by $\overline{\mathcal{P}}_{t}$ the set of (nonempty) overpartitions with parts less than or equal to $t$ and no parts equal to $t$ overlined, and by $\overline{\mathcal{G}}_{t}$ the set of (nonempty) overpartitions with the difference between largest and smallest parts at most $t$ and the largest part not overlined when the difference between largest and smallest parts is exactly $t$. Also, $\overline{\mathcal{B}}_{t}$ denotes the set of bipartitions where the first subpartition, which can be an empty partition, consists of only parts equal to $t$, none overlined, and the second subpartition is a nonempty overpartition with parts less than or equal to $t$.

The rest of this paper is organized as follows. In Section 2.1, we first construct a weight preserving map $\phi$ from $\overline{\mathcal{G}}_{t}$ to $\overline{\mathcal{P}}_{t}$. In Section 2.2 , we then construct another weight preserving map $\psi$ from $\overline{\mathcal{P}}_{t}$ to $\overline{\mathcal{B}}_{t}$. Finally, by combining these two maps, we will deduce that $\overline{\mathcal{G}}_{t}$ and $\overline{\mathcal{B}}_{t}$ have the same generating functions:

$$
\sum_{\pi \in \overline{\mathcal{G}}_{t}} z^{o(\pi)} q^{|\pi|}=\sum_{\beta \in \overline{\mathcal{B}}_{t}} z^{o(\beta)} q^{|\beta|},
$$

which is indeed equivalent to Theorem 1.1. In Section 3, a $q$-series proof of Theorem 1.1 will be given.

\section{A combinatorial approach}

2.1. Partition sets $\overline{\mathcal{G}}_{\boldsymbol{t}}$ and $\overline{\mathcal{P}}_{\boldsymbol{t}}$. For an overpartition $\pi=\left(\pi_{1}, \pi_{2}, \ldots, \pi_{\ell}\right)$ in $\overline{\mathcal{G}}_{t}$, let $s(\pi)=\left\lfloor\pi_{\ell} / t\right\rfloor$, where $\lfloor a\rfloor$ denotes the largest integer not exceeding $a$, and let 
$k(\pi)$ be the positive integer $k$ such that $\pi_{k} \geq(s(\pi)+1) t$ and $\pi_{k+1}<(s(\pi)+1) t$. If there is no such $k$, then we let $k(\pi)=0$.

We now define a map $\phi: \overline{\mathcal{G}}_{t} \rightarrow \overline{\mathcal{P}}_{t}$ as follows. For an overpartition $\pi \in \overline{\mathcal{G}}_{t}$, let $\ell(\pi)=\ell, s(\pi)=s$ and $k(\pi)=k$. Then

$$
\begin{aligned}
\phi: & \left(\pi_{1}, \pi_{2}, \ldots, \pi_{\ell}\right) \\
& \mapsto(\underbrace{t, t, t, \ldots, t}_{\substack{s(\ell-k)+(s+1) k \\
\text { times }}}, \pi_{k+1}-s t, \ldots, \pi_{\ell}-s t, \pi_{1}-(s+1) t, \ldots, \pi_{k}-(s+1) t),
\end{aligned}
$$

where all the parts equal to $t$ are not overlined, and if $\pi_{i}$ is overlined, then $\pi_{i}-s t$ (or $\pi_{i}-(s+1) t$ depending on the value of $i$ ) is overlined. In other words, $\phi$ takes $\pi$ to $\left(t, t, \ldots, t, a_{1}, \ldots, a_{\ell}\right)$ where $a_{1}, \ldots, a_{\ell}$ are $\pi_{1}, \ldots, \pi_{\ell}$ reduced modulo $t$, cyclically permuted to make them weakly decreasing.

Here we note that there may be parts equal to 0 in $\phi(\pi)$. If there are any parts equal to 0 , then we delete them so that $\phi(\pi)$ has positive parts only.

Theorem 2.1. $\phi$ is a weight preserving map from $\overline{\mathcal{G}}_{t}$ to $\overline{\mathcal{P}}_{t}$.

Proof. Since $\pi_{1}-\pi_{\ell} \leq t, s=\left\lfloor\pi_{\ell} / t\right\rfloor$, and $\pi_{k} \geq(s+1) t>\pi_{k+1}$, we have

$$
t>\pi_{k+1}-s t \geq \cdots \geq \pi_{\ell}-s t \geq \pi_{1}-(s+1) t \geq \cdots \geq \pi_{k}-(s+1) t .
$$

Thus the parts of $\phi(\pi)$ are less than or equal to $t$, and if there are overlined parts, they are less than $t$.

We now show that no more than one part of the same size is overlined. Since $\pi$ is an overpartition, at most one part of the same size is overlined in $\pi$. Hence, of $\pi_{1}-s t, \ldots, \pi_{k}-s t$, if there are overlined parts, then they must be of different sizes. For the same reason, of $\pi_{k+1}-(s+1) t, \ldots, \pi_{\ell}-(s+1) t$, overlined parts must be of different sizes. Thus, if $\pi_{\ell}-s t>\pi_{1}-(s+1) t$, then it is clear that all the overlined parts of $\phi(\pi)$ have different sizes.

Let us suppose that $\pi_{\ell}-s t=\pi_{1}-(s+1) t$. Then, we have $\pi_{1}-\pi_{\ell}=t$. By the definition of $\overline{\mathcal{G}}_{t}$, we know that all the parts equal to $\pi_{1}$ are not overlined. Thus, for parts in $\phi(\pi)$ that are equal to $\pi_{\ell}-s t=\pi_{1}-(s+1) t$, either the first occurrence or none may be overlined. Therefore, $\phi(\pi) \in \overline{\mathcal{P}}_{t}$.

We also note that the map $\phi$ preserves the weight of $\pi$, that is, $|\phi(\pi)|=|\pi|$.

As we see in the following example, the map $\phi$ is not a bijection.

Example 2.1. Let $t=3, \pi=(7, \overline{4})$ and $\tilde{\pi}=(\overline{4}, 4,3)$. Then

$$
\begin{aligned}
& s(\pi)=1, \quad k(\pi)=1, \quad \phi(\pi)=(3,3,3, \overline{1}, 1), \quad|\phi(\pi)|=|\pi|=11 ; \\
& s(\tilde{\pi})=1, \quad k(\tilde{\pi})=0, \quad \phi(\tilde{\pi})=(3,3,3, \overline{1}, 1), \quad|\phi(\tilde{\pi})|=|\tilde{\pi}|=11 .
\end{aligned}
$$

However, $\phi$ is a surjection since $\overline{\mathcal{P}}_{t}$ is a subset of $\overline{\mathcal{G}}_{t}$ and $\phi(\pi)=\pi$ for any $\pi \in \overline{\mathcal{P}}_{t}$. So, we will count how many pre-images each $\mu \in \overline{\mathcal{P}}_{t}$ has under $\phi$.

Let $\pi \in \overline{\mathcal{G}}_{t}$. We describe how to recover $\pi$ from $\phi(\pi)$. First, note that it is clear from the definition of $s(\pi)$ and $k(\pi)$ that $\pi_{i}-(s(\pi)+1) t$ and $\pi_{j}-s(\pi) t$ are the remainders of $\pi_{i}$ and $\pi_{j}$ when divided by $t$ for $1 \leq i \leq k(\pi)$ and $j>k(\pi)$. If the remainders are equal to 0 , then they are deleted in $\phi(\pi)$. Thus if we know the number of such deleted remainders, we can determine $\ell(\pi)$. Also, one of the deleted remainders may have been overlined.

We then need to find $s(\pi)$ and $k(\pi)$, where $s(\pi)$ is the quotient of the smallest part of $\pi$ when divided by $t$ and $k(\pi)$ counts the number of parts whose quotients 
are equal to $s(\pi)+1$. Therefore, once we have $\ell(\pi), k(\pi)$, and $s(\pi)$ along with the information on existence of an overlined deleted remainder, it is clear that we can recover $\pi$. Thus possible choices for $\ell(\pi), k(\pi)$, and $s(\pi)$ with having a deleted remainder overlined or not will determine the number of pre-images under $\phi$.

In the following lemma, we will see the range for $\ell(\pi)$. For any $\mu \in \overline{\mathcal{P}}_{t}$, we use $m(\mu)=m_{t}(\mu)$ to count the number of parts of $\mu$ equal to $t$.

Lemma 2.2. Let $\pi$ be a nonempty overpartition in $\overline{\mathcal{G}}_{t}$ and $\mu=\phi(\pi)$ in $\overline{\mathcal{P}}_{t}$. Then we have

(i) $\ell(\pi) \leq \ell(\mu)$;

(ii) $\ell(\pi) \geq \ell(\mu)-m(\mu)+\delta_{\ell(\mu), m(\mu)}$, where $\delta_{\ell(\mu), m(\mu)}$ is the Kronecker delta.

Proof. First, (i) is almost trivial. Under $\phi$, each part of $\pi$ splits into its residue modulo $t$ and as many $t$ 's as the quotient, i.e., each part $\pi_{i}$ contributes $\left\lceil\pi_{i} / t\right\rceil$ to the number of parts of $\mu$. Thus $\ell(\pi) \leq \ell(\mu)$.

Next, we prove (ii). If all of the parts of $\mu$ are $t$, i.e., $\ell(\mu)=m(\mu)$, then

$$
\ell(\mu)-m(\mu)+\delta_{\ell(\mu), m(\mu)}=1 \leq \ell,
$$

where the last inequality follows from the fact that $\pi$ is nonempty.

We now suppose that $\mu$ has a part not equal to $t$, i.e, $\ell(\mu)-m(\mu) \geq 1$. From the definition of $\phi$, we know that the parts of $\mu$ not equal to $t$ are the positive remainders of the parts of $\pi$, so at most $\ell$ parts of $\mu$ are not equal to $t$. Hence

$$
\ell(\mu)-m(\mu)+\delta_{\ell(\mu), m(\mu)}=\ell(\mu)-m(\mu) \leq \ell .
$$

This completes the proof of (ii).

It follows from Lemma 2.2 that

$$
\delta_{\ell(\mu), m(\mu)} \leq \ell(\pi)-(\ell(\mu)-m(\mu)) \leq m(\mu),
$$

where $\ell(\pi)-(\ell(\mu)-m(\mu))$ is the number of multiples of $t$ in $\pi$.

Lemma 2.3. Let $n$ be a fixed positive integer, and $n^{\prime}$ a fixed nonnegative integer. Then the following system of equations

$$
\begin{cases}x+y & =n, \\ s x+(s+1) y & =n^{\prime}\end{cases}
$$

has exactly one simultaneous solution $(x, y, s) \in \mathbb{Z}_{>0} \times \mathbb{Z}_{\geq 0} \times \mathbb{Z}_{\geq 0}$.

Proof. We readily see that $y=n^{\prime}-s n$. Also, since $x>0$ and $y \geq 0$, it follows from the first equation that $0 \leq y<n$. Hence

$$
\frac{n^{\prime}}{n}-1<s \leq \frac{n^{\prime}}{n},
$$

from which it follows that $s=\left\lfloor n^{\prime} / n\right\rfloor$. Therefore, there is only one solution $(x, y, s)$.

We are now ready to determine how many pre-images an overpartition in $\overline{\mathcal{P}}_{t}$ has.

Theorem 2.4. Let $\mu$ be a nonempty overpartition in $\overline{\mathcal{P}}_{t}$.

(i) If $\ell(\mu)=m(\mu)$, then there are exactly $2 m(\mu)$ pre-images in $\overline{\mathcal{G}}_{t}$ under $\phi$. Moreover, of those pre-images, exactly $m(\mu)$ pre-images have no overlined parts, and the other $m(\mu)$ pre-images have the first occurrence of the smallest parts overlined. 
(ii) If $\ell(\mu)>m(\mu)$, then there are exactly $2 m(\mu)+1$ pre-images in $\overline{\mathcal{G}}_{t}$ under $\phi$. Moreover, of those pre-images, exactly $m(\mu)+1$ pre-images have the same number of overlined parts as $\mu$ and the other $m(\mu)$ pre-images have one more overlined part than $\mu$ does.

Proof. Let $\pi$ be a pre-image of $\mu$. By Lemma 2.2, we know that

$$
\ell(\mu)-m(\mu)+\delta_{\ell(\mu), m(\mu)} \leq \ell(\pi) \leq \ell(\mu) .
$$

Hence, for any integer $\ell$ in this range, we want to know how many $\pi \in \overline{\mathcal{G}}_{t}$ with $\ell(\pi)=\ell$ can be pre-images of $\mu$.

In order for $\pi$ to be a pre-image of $\mu$ with $\ell(\pi)=\ell, s(\pi)$ and $k(\pi)$ must satisfy

$$
s(\pi)(\ell-k(\pi))+(s(\pi)+1) k(\pi)=m(\mu) .
$$

By the definition of $k(\pi)$, it should be less than $\ell(\pi)$, i.e., $\ell-k(\pi)>0$. Thus, (2.4) is equivalent to that $(\ell-k(\pi), k(\pi), s(\pi))$ is a solution to $(2.2)$ with $n=\ell$ and $n^{\prime}=m(\mu)$, which is unique.

(i) Suppose that $\ell(\mu)=m(\mu)$. By $(2.3)$, there are $m(\mu)$ choices for $\ell$. For a fixed $\ell, k(\pi)$ and $s(\pi)$ are uniquely determined as seen above. With these $(\ell, k(\pi), s(\pi))$, we can construct $\pi$, in which parts are multiples of $t$ differing by at most $t$ and there are no overlined parts.

For each $\pi$, by having the first occurrence of the smallest parts overlined, we obtain a different pre-image. Therefore, the total number of pre-images must be equal to $2 m(\mu)$ as claimed. Also, $m(\mu)$ pre-images have no overlined parts and the other $m(\mu)$ pre-images have one overlined smallest part.

(ii) Suppose that $\ell(\mu)>m(\mu)$. By (2.3), there are $(m(\mu)+1)$ choices for $\ell$. For a fixed $\ell, k(\pi)$ and $s(\pi)$ are uniquely determined. With these $(\ell, k(\pi), s(\pi))$, we can construct $\pi$, in which no multiples of $t$ are overlined.

Note that from the construction of $\phi, \ell(\mu)-m(\mu)$ counts the nonzero residues of the parts of $\pi$ modulo $t$. So, if $\ell(\pi)>\ell(\mu)-m(\mu)$, then $\pi$ must have multiples of $t$ as parts. For such $\pi$, by having the first occurrence of the smallest multiples of $t$ overlined, we obtain a different pre-image.

Therefore, the total number of pre-images must be equal to $(2 m(\mu)+1)$ as claimed. Also, $(m(\mu)+1)$ pre-images have the same number of overlined parts as $\mu$ and the other $m(\mu)$ pre-images have one more overlined part than $\mu$ does.

Theorem 2.4 yields

$$
\sum_{\pi \in \overline{\mathcal{G}}_{t}} z^{o(\pi)} q^{|\pi|}=\sum_{\mu \in \overline{\mathcal{P}}_{t}}\left(\left(1-\delta_{\ell(\mu), m(\mu)}\right)+(1+z) m(\mu)\right) z^{o(\mu)} q^{|\mu|} .
$$

In the following example, we present how to find all the pre-images $\pi$ of $\mu$.

Example 2.2. Let $t=3$.

(i) Let $\mu=(3,3,3)$. Since $\ell(\mu)=m(\mu)=3$, by Lemma 2.2

$$
1 \leq \ell(\pi) \leq 3
$$

By solving (2.4), we have $(\ell(\pi), k(\pi), s(\pi))=(1,0,3),(2,1,1),(3,0,1)$, which yield

$$
(6,3),(6, \overline{3})
$$


respectively. There are $2 m(\mu)$ pre-images.

(ii) Let $\mu=(3,3,3, \overline{1}, 1)$. Since $\ell(\mu)=5$ and $m(\mu)=3$, by Lemma 2.2

$$
2 \leq \ell(\pi) \leq 5 .
$$

By solving (2.4), we have $(\ell(\pi), k(\pi), s(\pi))=(2,1,1),(3,0,1),(4,3,0),(5,3,0)$, which yield

$(3,3,3, \overline{1}, 1),(\overline{3}, 3,3, \overline{1}, 1)$,

respectively. Thus, there are $2 m(\mu)+1$ pre-images.

2.2. Partition sets $\overline{\mathcal{P}}_{t}$ and $\overline{\mathcal{B}}_{t}$. Let us recall the definition of $\overline{\mathcal{B}}_{t}$, from which it is clear that

$$
\begin{aligned}
\sum_{\beta \in \overline{\mathcal{B}}_{t}} z^{o(\beta)} q^{|\beta|} & =\left(1+q^{t}+q^{2 t}+\cdots\right)\left(\frac{(-z q)_{t}}{(q)_{t}}-1\right) \\
& =\frac{1}{1-q^{t}}\left(\frac{(-z q)_{t}}{(q)_{t}}-1\right),
\end{aligned}
$$

where $o(\beta)$ denotes the number of overlined parts in $\beta$, which is indeed the number of overlined parts in the second subpartition of $\beta$.

We now construct a map $\psi: \overline{\mathcal{B}}_{t} \rightarrow \overline{\mathcal{P}}_{t}$ as follows:

(1) First collect all parts equal to $t$ in both subpartitions and replace an overlined $t$ by a non-overlined $t$;

(2) and then append the remaining parts in the second subpartition to the parts collected in (1).

For example, $[(3),(3,3, \overline{1}, 1)]$ and $[(3),(\overline{3}, 3, \overline{1}, 1)]$ are both mapped to $(3,3,3, \overline{1}, 1)$ under $\psi$.

Let $\mu \in \overline{\mathcal{P}}_{t}$. Suppose that $\ell(\mu)=m(\mu)$, i.e., $\mu$ has parts equal to $t$ only. Then, its pre-image $\beta$ must be a bipartition of this form

$$
[(\underbrace{t, \ldots, t}_{m(\mu)-x}),(\underbrace{t, \ldots, t}_{x})]
$$

for some $x>0$ with either the first occurrence or none of $t$ 's in the second subpartition overlined. Thus there are $2 m(\mu)$ pre-images of $\mu$ in $\overline{\mathcal{B}}_{t}$ under $\psi$. Of those pre-images, $m(\mu)$ pre-images have the same number of overlined parts as $\mu$, and the other $m(\mu)$ pre-images have one more overlined part than $\mu$.

Suppose that $\ell(\mu)>m(\mu)$, i.e., $\mu$ has a part not equal to $t$. Then, its pre-image $\pi$ must be a bipartition of this form

$$
\left[(\underbrace{t, \ldots, t}_{m(\mu)-x}),(\underbrace{t, \ldots, t}_{x}, \mu_{m(\mu)+1}, \ldots)\right]
$$

for some $x \geq 0$ with either the first occurrence or none of $t$ 's in the second subpartition overlined. Thus there are $2 m(\mu)+1$ pre-images of $\mu$ in $\overline{\mathcal{B}}_{t}$ under $\psi$. Of those pre-images, $(m(\mu)+1)$ pre-images have the same number of overlined parts as $\mu$, and the other $m(\mu)$ pre-images have one more overlined part than $\mu$. 
Therefore, it follows from the map $\psi$ that

$$
\sum_{\mu \in \overline{\mathcal{P}}_{t}}\left(\left(1-\delta_{\ell(\mu), m(\mu)}\right)+(1+z) m(\mu)\right) z^{o(\mu)} q^{|\mu|}=\sum_{\beta \in \overline{\mathcal{B}}_{t}} z^{o(\beta)} q^{|\beta|} .
$$

By $(2.5),(2.6)$, and $(2.7)$,

$$
\sum_{n \geq 1} \sum_{m \geq 0} g_{t}(m, n) z^{m} q^{n}=\sum_{\pi \in \overline{\mathcal{G}}_{t}} z^{o(\pi)} q^{|\pi|}=\sum_{\beta \in \overline{\mathcal{B}}_{t}} z^{o(\beta)} q^{|\beta|}=\frac{1}{1-q^{t}}\left(\frac{(-z q)_{t}}{(q)_{t}}-1\right)
$$

which completes the proof of Theorem 1.1.

\section{Final remarks}

We remark that, by slightly modifying the first proof of [5, Theorem 2.1], we can also prove Theorem 1.1 analytically.

Let

${ }_{r+1} \phi_{s}\left(\begin{array}{c}a_{0}, a_{1}, a_{2} \ldots, a_{r} \\ b_{1}, b_{2}, \ldots, b_{s}\end{array} ;, z\right):=\sum_{n \geq 0} \frac{\left(a_{0} ; q\right)_{n}\left(a_{1} ; q\right)_{n} \cdots\left(a_{r} ; q\right)_{n}}{(q ; q)_{n}\left(b_{1} ; q\right)_{n} \cdots\left(b_{s} ; q\right)_{n}}\left((-1)^{n} q^{\left(\begin{array}{c}n \\ 2\end{array}\right)}\right)^{s-r} z^{n}$.

Then we will need the following identities later.

Lemma 3.1 (First $q$-Chu-Vandermonde Sum [1, Eq. (17.6.2)]). We have

$$
{ }_{2} \phi_{1}\left(\begin{array}{c}
a, q^{-n} \\
c
\end{array} ; q, c q^{n} / a\right)=\frac{(c / a ; q)_{n}}{(c ; q)_{n}} .
$$

Lemma 3.2 ([1, Eq. (17.9.6)]). We have

$$
{ }_{3} \phi_{2}\left(\begin{array}{c}
a, b, c \\
d, e
\end{array} ; q, d e /(a b c)\right)=\frac{(e / a ; q)_{\infty}(d e /(b c) ; q)_{\infty}}{(e ; q)_{\infty}(d e /(a b c) ; q)_{\infty}}{ }_{3} \phi_{2}\left(\begin{array}{l}
a, d / b, d / c \\
d, d e /(b c)
\end{array} ; q, e / a\right) .
$$

First, note that the generating function for partitions in $\overline{\mathcal{G}}_{t}$ with smallest part equal to $r$ is

$$
\frac{(1+z) q^{r}}{1-q^{r}} \frac{1+z q^{r+1}}{1-q^{r+1}} \cdots \frac{1+z q^{r+t-1}}{1-q^{r+t-1}} \frac{1}{1-q^{r+t}},
$$

in which the coefficient of $z^{m} q^{n}$ counts the number of such overpartitions of $n$ with exactly $m$ overlined parts. Hence

$$
\begin{aligned}
\sum_{n \geq 1} \sum_{m \geq 0} g_{t}(m, n) z^{m} q^{n} & =\sum_{r \geq 1} \frac{(1+z) q^{r}}{1-q^{r}} \frac{1+z q^{r+1}}{1-q^{r+1}} \cdots \frac{1+z q^{r+t-1}}{1-q^{r+t-1}} \frac{1}{1-q^{r+t}} \\
& =(1+z) \sum_{r \geq 1} \frac{(q)_{r-1}(-z q)_{r+t-1}}{(q)_{r+t}(-z q)_{r}} q^{r} \\
& =(1+z) q \sum_{r \geq 0} \frac{(q)_{r}(-z q)_{r+t}}{(q)_{r+t+1}(-z q)_{r+1}} q^{r} \\
& =\frac{(1+z) q(-z q)_{t}}{(1+z q)(q)_{t+1}} \sum_{r \geq 0} \frac{(q)_{r}(q)_{r}\left(-z q^{t+1}\right)_{r}}{(q)_{r}\left(q^{t+2}\right)_{r}\left(-z q^{2}\right)_{r}} q^{r} \\
& =\frac{(1+z) q(-z q)_{t}}{(1+z q)(q)_{t+1}}{ }_{3} \phi_{2}\left(\begin{array}{c}
q, q,-z q^{t+1} \\
-z q^{2}, q^{t+2}
\end{array} ;, q\right)
\end{aligned}
$$




$$
\begin{aligned}
& =\frac{(1+z) q(-z q)_{t}}{(1+z q)(q)_{t+1}} \frac{\left(q^{t+1}\right)_{\infty}\left(q^{2}\right)_{\infty}}{\left(q^{t+2}\right)_{\infty}(q)_{\infty}}{ }_{3} \phi_{2}\left(\begin{array}{c}
q,-z q, q^{1-t} \\
-z q^{2}, q^{2} \\
\text { (by Eq. (3.2)) }
\end{array} ;, q^{t+1}\right) \\
& =\frac{(1+z) q(-z q)_{t}}{(1-q)(1+z q)(q)_{t}} \sum_{r \geq 0} \frac{(-z q)_{r}\left(q^{1-t}\right)_{r}}{\left(-z q^{2}\right)_{r}\left(q^{2}\right)_{r}} q^{r(t+1)} \\
& =-\frac{(-z q)_{t}}{\left(1-q^{t}\right)(q)_{t}} \sum_{r \geq 0} \frac{(-z)_{r+1}\left(q^{-t}\right)_{r+1}}{(-z q)_{r+1}(q)_{r+1}} q^{(r+1)(t+1)} \\
& =-\frac{(-z q)_{t}}{\left(1-q^{t}\right)(q)_{t}}\left({ }_{2} \phi_{1}\left(-z, q^{-t} ; q, q^{t+1}\right)-1\right) \\
& =-\frac{(-z q)_{t}}{\left(1-q^{t}\right)(q)_{t}}\left(\frac{(q)_{t}}{(-z q)_{t}}-1\right) \\
& =\frac{1}{1-q^{t}}\left(\frac{(-z q)_{t}}{(q)_{t}}-1\right) .
\end{aligned}
$$

Acknowledgements. We thank the referees for their careful reading and helpful comments. The second author was partially supported by a grant (\#280903) from the Simons Foundation.

\section{References}

1. G. E. Andrews, $q$-Hypergeometric and related functions, NIST handbook of mathematical functions, 419-433, U.S. Dept. Commerce, Washington, DC, 2010.

2. G. E. Andrews, M. Beck, and N. Robbins, Partitions with fixed differences between largest and smallest parts, Proc. Amer. Math. Soc. 143 (2015), no. 10, 4283-4289.

3. F. Breuer and B. Kronholm, A polyhedral model of partitions with bounded differences and a bijective proof of a theorem of Andrews, Beck, and Robbins, Res. Number Theory 2 (2016), Art. 2, 15 pp.

4. R. Chapman, Partitions with bounded differences between largest and smallest parts, Australas. J. Combin. 64 (2016), 376-378.

5. S. Chern, An overpartition analogue of partitions with bounded differences between largest and smallest parts, Discrete Math. 340 (2017), no. 12, 2834-2839.

6. J. Dousse and B. Kim, An overpartition analogue of the $q$-binomial coefficients, Ramanujan J. 42 (2017), no. 2, 267-283.

(S. Chern) Department of Mathematics, The Pennsylvania State University, UniverSITY PARK, PA 16802, USA

E-mail address: shanechern@psu.edu; chenxiaohang92@gmail.com

(A. J. Yee) Department of Mathematics, The Pennsylvania State University, UniverSITY PARK, PA 16802, USA

E-mail address: auy2@psu.edu 